\title{
Présentation
}

\section{Migrants, réfugiés, la politique interroge la clinique}

\author{
[ Bertrand Piret ${ }^{[1]}$, Olivier Douville ${ }^{[2]}$
}

e numéro répond à la demande que Psychologie Clinique fit à l'équipe de Parole sans frontière de coordonner un dossier consacré à de nouveaux aspects de la clinique de l'exil, marqués des ruptures violentes d'avec le sol natal. C'est l'occasion de faire le point sur cette clinique à partir de l'expérience de Parole sans frontière. Ce dispositif particulier, dont la mise en place remonte à 24 ans à Strasbourg, a évolué avec d'autres centres comme l'équipe du Centre de Santé Mentale Ulysse, à Bruxelles. Ils ont pour objet l'accueil et la prise en charge globale des personnes exilées, en précarité de séjour et en souffrance psychologique, quels que soient leur pays d'origine, leurs appartenances culturelles et les raisons de leur exil. La mondialisation du marché, les violences des conflits et des guerres civiles, sont deux facteurs propices à la circulation des personnes hors de leur pays d'origine, à la recherche d'un lieu d'asile où la survie est possible.

On mesure alors que les enjeux de la clinique sont interrogés par la politique. Les dispositifs cliniques sont aussi tributaires de la façon dont les thérapeutes et les autres professionnels qui y travaillent prennent position par rapport aux discours dominants (à propos de l'immigration, des réfugiés, des "sans-papiers ", de la précarité, etc.) et sanitaires (à propos de la manière dont est désormais définie la santé mentale et les critères de normativité qui redéfinissent la souffrance sociale et la souffrance psychique).

Les cliniques de l'exil deviennent de plus en plus des cliniques qui rencontrent la réalité des exclusions et des déracinements violents. Elles ne sauraient se réduire à une clinique strictement inter ou intra-culturelle. Infléchir la notion d'écart culturel ou d'étrangeté culturelle à toute rencontre entre un dispositif de santé et les

[1] Psychiatre, psychanalyste, 5 rue Grandidier 67000 Strasbourg.

[2] Psychanalyse, Psychologue clinicien (E.P.S. de Ville-Évrard, 93330 Neuilly/Marne), Maître de conférences hors classe des Universités. Association Française des Anthropologues. Laboratoire CRPMS (Université Paris Diderot-Paris 7). 
personnes dites “ étrangères " qui lui sont adressées, revient à méconnaître avec un certain acharnement la façon dont les modes de vie, d'espérer, voire de souffrir de bien des exilés ne se réduisent pas à des compromis entre modernité et tradition, mais renvoient à la dimension de la légitimité d'exister et de trouver des points d'accueil dans nos institutions.

Ce numéro a l'ambition de maintenir vivante la prise en compte de l'inconscient dans cette clinique. Il fallait alors ouvrir des débats avec la façon dont sont pensés et mis en ouvre des dispositifs. Comment l'inconscient se faufile-t-il dans des situations d'interlangues? Comment travailler avec des interprètes?

Sevdalina Todorova prête avec bien d'autres interprètes l'écoute sensible qui permet le travail psychothérapique « à trois ». Andrée Bauer fut à la fois pendant plusieurs années un pilier du bureau de l'association et une référente au niveau clinique. Nicolas Velut a travaillé à nos côtés pendant son passage à Strasbourg avant de développer ses activités à Toulouse. Il est un de fidèles partenaires de Parole sans frontière comme l'est aussi Pascale De Ridder, qui anime avec ses collègues bruxellois le Centre de soins et d'accompagnement de l'association Ulysse, en compagnie désormais de Cihan Gunes, psychologue clinicienne qui auparavant travailla à Parole sans frontière. Jean-Christophe Weber quant à lui, Professeur de médecine et chef de pôle aux Hôpitaux Universitaires de Strasbourg est un précieux soutien tant par l'appui aux dispositifs d'interprétariat en médecine qu'il promeut au sein de l'hôpital qu'à travers la profondeur et la pertinence de ses recherches dans le domaine de l'éthique, de la relation médecin malade et des implications dans ce champ des outils fournis par la psychanalyse.

Tels furent les apports du Centre Paroles sans frontière. D'autres contributeurs ont par la suite spontanément apporté des travaux sur l'exclusion et la place du soin psychique dans les dispositifs de travail social en direction des grands exclus (Olivier Jan, Lara Pennec), sur les incidences des déshumanisations dues aux violences économiques et politiques (Marina Koussouri et Brigitte Haie). Saverio Tomsella, Janine Altounian et Sébastien Talon permettent aussi de penser comment, au-delà des catastrophes subjectives liées aux traumatismes collectifs, la vie psychique reprend goût à la durée et à la transmission. Accompagner et aider une personne à la reconstruction des figures d'altérité et des topos de dialogue est certes mettre en œuvre un processus thérapeutique, mais c'est aussi s'engager dans le sens d'une résistance à ces montées en puissance des rétrécissements identitaires et à la généralisation des clivages entre nous et les autres, à la passion grandissante pour les frontières.

C'est bien parce que le politique travaille et met à mal l'intime que la reconstruction d'un espace d'interlocution psychique, entre la personne et ses thérapeutes, mais avec des altérités internes, est un mouvement de refus de la banalisation des exclusions matérielles et psychiques d'un nombre croissant de femmes, d'hommes et d'enfants. Certaines mises à nu de la condition humaine sont là pour nous rappeler ce qu'une clinique par trop élégiaque veut oublier: il y a des effets du politique dans la vie psychique. Ce dont ce numéro témoigne. 